\title{
CONDICIONAR, FOCALIZAR E INVERTIR EN CAPITAL HUMANO: EL PROGRAMA "FAMILIAS EN ACCIÓN" O LA RECETA INTERNACIONAL DE LUCHA CONTRA LA POBREZA PARA COLOMBIA
}

Valeria Ayola-Betancourt École de Hautes Études en Sciences Sociales (EHESS) Francia 
Panorama Económico, Vol. 26 - No.1(Enero - Marzo de 2018), pp. 37-58

Valeria Ayola-Betancourt

\title{
Conditioning, targeting and investing in human capital: The "Familias en Acción" CCT or the international fight against poverty recipe for Colombia
}

\begin{abstract}
Resumen
El presente artículo pone en contexto la construcción del paradigma de la lucha contra la pobreza en la agenda internacional, como marco para la implementación de programas de transferencias condicionadas (PTC) en países en desarrollo. Se plantea una reconstrucción teórica de los fundamentos conceptuales reunidos en la fórmula de condicionar, focalizar e invertir en capital humano, como elementos técnicos que permiten el posicionamiento de estos programas en la política de asistencia social de los países de América Latina. Finalmente, a partir del caso colombiano, y los resultados del PTC "Familias en Acción" establecen el consenso científico y político a su favor. En éste sentido, se propone una reflexión teórica y crítica, en el cruce de la sociología de la desigualdad y la economía del desarrollo.

Palabras clave: Pobreza, Desigualdad, Desarrollo, Transferencias Monetarias Condicionadas, Política Social. Clasificación JEL: A14, I32, I38, O19, Z18
\end{abstract}

\section{Conditioning, targeting and investing in human capital: The "Familias en Acción" CCT or the international fight against poverty recipe for Colombia}

\begin{abstract}
This article contextualize the construction of the paradigm of poverty reduction in the international agenda, as a determining framework for the implementation of Conditional Cash Transfer Programs (CCT) in developing countries. It is proposed a theoretical reconstruction of its conceptual framework in line of conditioning, targeting and investing in human capital, as key elements for their implementation in Latin America as the most suitable and recommended instrument for the reduction of poverty in the region. Finally, in the light of the Colombian case and its "Familias en Acción" CCT Program results, set scientific and political consensus in its favor. By this, it is presented a theoretical and critical reflection about poverty and the development discourse at the junction of the sociology of inequality and development economics Keywords: Poverty, inequality, development, social policy, conditional cash transfers.
\end{abstract}

JEL Classification: A14, I32, I38, O19, Z18

\section{Conditionner, cibler et investir dans le capital humain: Le programme "Familias en Acción" ou la recette internationale de lutte contre la pauvreté pour la Colombie}

\footnotetext{
Résumé

Cet article contextualise la construction du paradigme de la réduction de la pauvreté dans l'agenda international, en tant que cadre déterminant pour la mise en œuvre de programmes de transferts monétaires conditionnels (CCT) dans les pays en développement. Il est proposé de reconstruire théoriquement son cadre conceptuel en matière de conditionnement, de ciblage et d'investissement dans le capital humain, en tant qu'éléments essentiels de leur mise en œuvre en Amérique latine comme un instrument le plus approprié et recommandé pour réduire la pauvreté dans la région. Enfin, à la lumière du cas colombien et des résultats de son programme CCT "Familias en Acción", a mis le consensus scientifique et politique en sa faveur. En somme, il est présenté une réflexion théorique et critique sur la pauvreté et le discours du développement à la croisée de la sociologie de l'inégalité et de l'économie du développement..

Mots-clés: Pauvreté, Inégalités, Développement, Politique Sociale, Transferts Monétaires Conditionnels.

Nomenclature JEL: I A14, I32, I38, O19, Z18
} 


\section{Condicionar, focalizar e invertir en capital humano. El programa "Familias en Acción" o la receta internacional de lucha contra la pobreza para Colombia}

INFORMACIÓN DEL ARTÍCULO

Recepción de artículo: 22/03/2017

Concepto de evaluación: 02/06/2017

Aceptación de artículo: 18/08/2017
Valeria Ayola-Betancourt École de Hautes Études en Sciences Sociales (EHESS)

Francia

Este artículo pone en contexto la construcción del paradigma de la Lucha Contra la Pobreza (LCP) en la agenda internacional, como marco determinante para la implementación de los Programas de Transferencias Condicionadas (PTC) en los Países en Desarrollo (PED). En este artículo, se emprende una reconstrucción conceptual de los fundamentos teóricos de los PTC, lo cual permite vincular el discurso de organizaciones internacionales como el Banco Mundial (BM) -como espacio de producción y difusión de conocimiento-, con postulados y teorías elaboradas desde la rama de la economía del desarrollo.

De esta forma, se estudia el ámbito global como contexto determinante para la consolidación del Programa Familias en Acción (PFA) en Colombia, el cual será puesto en marcha en el año 2001 y se convertirá en ley de la república en el año 2012 (Ley 1532 del 2012). Esta inscripción como política de Estado y ya no de gobierno lo consolida como pilar de la política de asistencia social ${ }^{1}$ y como instrumento por excelencia de la lucha contra la pobreza en Colombia.

Este documento se propone contribuir a la reflexión teórica sobre los temas de pobreza y desigualdad, es una investigación analítica-descriptiva donde se realiza un análisis del discurso y una revisión bibliográfica de fuentes y datos secundarios. Conviene mencionar que este documento es una prolongación de una tesis de maestría (2015) la

1 PFA constituye el programa faro de la política social colombiana, el gasto en PTC en Colombia constituye casi una cuarta parte del gasto en protección social, de hecho, 22,6\% del gasto en protección social (no contributivo) es destinado a este programa. En 2015 representaba 0,3\% del PIB (CEPAL, 2017).

* Autor para correspondencia

Correos electrónicos: valeria.ayola@ehess.fr 
cual incluyó un componente empírico en el departamento de Bolívar y donde se estudió la apropiación del PFA por parte de los beneficiarios (Ayola Betancourt, 2016).

Este artículo se estructura de la siguiente forma: primero, se describe la construcción del objeto de estudio desde un enfoque sociológico. Segundo, se analiza la dinámica de la regulación de la pobreza contemporánea en los PED, como estando en el cruce de la política social nacional y de la internacional LCP. Tercero, se contextualiza la entrada del paradigma de la LCP y la transformación del discurso del BM en los años noventa y dos mil. Cuarto, se relacionan los conceptos teóricos que fundamentan los PTC como instrumentos técnicos de la LCP. Finalmente, a la luz del caso colombiano y del PTC Familias en Acción, se confrontan los resultados del programa con algunos datos e indicadores que cuestionan el consenso científico y político a su favor.

\section{DE LAS REPRESENTACIONES SOCIALES AL OBJETO DE ESTUDIO}

Alrededor del programa FA se han forjado un conjunto de imaginarios y representaciones relacionados con su funcionamiento y sus efectos en los beneficiarios. Al mismo tiempo, con el programa se han revivido viejas representaciones sociales asociadas a la pobreza.

Basado en el terreno realizado, rápidamente se puede decir que para los colombianos el programa se presenta como un programa asistencialista, el cual incentiva la pereza de sus beneficiarios ${ }^{2}$, beneficia a familias que no lo necesitan y genera un aumento en la tasa de natalidad de sus beneficiarios. En cuanto a representaciones de corte político, el programa no ha logrado deshacerse de los dirigentes que lo popularizaron, en efecto, FA es visto como el programa social del expresidente Álvaro Uribe (2002-2010) y en menor medida de su sucesor J.M. Santos (2010-2018) .

Las representaciones ordinarias son importantes para la investigación científica, estas como hechos sociales son un reflejo de la manera como los individuos aprehenden los fenómenos sociales a la luz de su experiencia y condición socioeconómica. Existe así, una dificultad por parte de los ciudadanos en el reconocimiento del programa FA como estrategia idónea de lucha contra la pobreza. ¿Cómo explicar que un Estado con una política social históricamente minimalista haya finalmente adoptado un programa articulado destinado a los más pobres? ¿Cómo entender la relación de asistencia que propone el programa en el contexto laboral colombiano? ¿Cómo explicar que se adopte en Colombia un subsidio en efectivo y sin condicionalidad en el uso-gasto del mismo? ¿Cómo explicar que unos sean beneficiarios y el resto no?

Algunas de estas preguntas se responden en este artículo, sin embargo lo sociológicamente interesante es el desfase existente entre la representación ordinaria y el instrumento como construcción técnica y científica. Todo esto, ha dificultado la comprensión del programa desde su dimensión internacional, científica y normativa. Tarea a la que se propone

2 De acuerdo a la encuesta Barómetro de las Américas o LAPOP realizada en 2012, 45.7\% de los encuestados piensa que la asistencia pública genera pereza en sus beneficiarios (LAPOP, 2012). El mismo informe describe una correlación entre esta respuesta y la pertenencia a los quintes de ingresos 4 y 5.

3 Esto, nos remite a la función política que ha tenido este programa en las pasadas administraciones con algunos efectos electorales (Pulido, 2013), efecto que el legislador quiso limitar al inscribir FA en el marco legal colombiano a través de la ley 1532 de 2012. 
contribuir este artículo, el cual plantea un debate desde la sociología de la desigualdad a la economía del desarrollo.

\section{LA REGULACIÓN DE LA POBREZA CONTEMPORÁNEA: ENTRE POLÍTICAS NACIONALES Y REFERENTES GLOBALES.}

La regulación de la pobreza involucra al conjunto de iniciativas privadas y públicas ${ }^{4}$ que buscan mitigar las formas extremas de la pobreza en un contexto dado. Analizar la regulación de la pobreza contemporánea, nos demanda el estudio de las estrategias nacionales a la luz de los referentes supranacionales que las influencian.

Una de las formas que toma la regulación de la pobreza en los Estados occidentales en el siglo $\mathrm{XX}$, es un arreglo institucional conocido como la política social. Esta es una herramienta de carácter distributivo que emana del poder político con efectos económicos y sociales. Su existencia garantiza el goce de los derechos sociales por parte de los ciudadanos, al mismo tiempo que mitiga las manifestaciones extremas de la diferencia entre los miembros de una sociedad y los efectos de las crisis económicas. Los Estados Providencia o welfare states europeos de la segunda mitad del siglo XX, inspiraron la constitución de los sistemas de seguridad social en América Latina, dentro de los cuales podemos resaltar los casos de Uruguay y Argentina.

La política social se compone por un lado, de la política de protección y seguridad social, la cual protege al trabajador en el marco de la relación laboral (riesgos profesionales, pensiones) y frente a los riesgos e incertidumbres de la vida (enfermedad, desempleo) 5 . Por otro lado, la política de asistencia social comprende al conjunto de programas destinados a quienes se encuentran fuera de la esfera laboral pero que por su condición de debilidad manifiesta requieren asistencia, de alguna forma es una política transversal que puede tener como objeto todos los grupos etarios y concentra lo que otrora era el campo de la caridad o de la asistencia a pobres y desvalidos.

En esta sección se postula que con la implementación de nuevos instrumentos de lucha contra la pobreza en la primera década del siglo XXI se asiste a un cambio en la regulación de la pobreza en los PED. Transformación donde los referentes nacionales y en particular la perspectiva universalista de los derechos sociales, cede ante nuevas modalidades de intervención promovidas y financiadas por los organismos económicos internacionales. Esta confluencia no siempre tuvo lugar, de hecho, desde el BM se buscó atacar la pobreza de los países en desarrollo a través de la financiación de grandes obras de infraestructura y de la inversión en capital. La fórmula era sencilla: crecimiento económico era sinónimo de desarrollo en un país. A lo cual se suma una visión simplificada de la modernización, aplicada por igual a África, Asia y América Latina.

4 En su ámbito estatal se conoce como política de asistencia social, pero se trata de una esfera que ha visto grandes transformaciones en el tiempo. Esta puede realizarse sin intermediarios (clan, comunidad, familia) o a través de diferentes arreglos institucionales o morales (Iglesia hasta bien entrado el S.XX). Se trata de la distinción entre solidaridad mecánica y orgánica propuesta por Durkheim en 1893.

$5 \mathrm{La}$ adopción de los diferentes modelos de Estado-providencia en la Europa de la postguerra son el reflejo de la manera en que las sociedades occidentales materializaron su ideal de justicia social en concordancia con los principios que las sustentan. Donde se evidencia la dualidad entre los principios de igualdad-libertad, privatizaciónmanejo estatal, universalidad-focalización, entre otros. 
Asimismo, en el marco de los planes de ajuste estructural (PAE) introducidos desde los años setenta, las instituciones de Breton Woods promoverán el desmonte de los sistemas de protección social nacionales en los PED por considerarlos opciones costosas para un contexto de escasez de recursos. Así, la protección social aparecía como poco recomendable para el tercer mundo.

El argumento anterior será reconsiderado solo hasta los años 2000, gracias a la presión que realizarán diferentes organizaciones como la Organización Internacional del Trabajo (OIT), la Agencia de Desarrollo del Reino Unido, el Programa de Naciones Unidas para el Desarrollo (PNUD) así como diversas ONG's (Merrien, 2013). De esta forma la protección social será poco a poco reconocida por el BM como instrumento de lucha contra la pobreza y de desarrollo. Sin embargo, como se mencionará ulteriormente en la sección 3.1 no será bajo los mismos principios de solidaridad e igualdad del estado providencia sino bajo el enfoque del Manejo social del riesgo.

\section{DE LA LUCHA CONTRA LA POBREZA A LOS PROGRAMAS DE TRANSFERENCIAS CONDICIONADAS.}

En la década de los noventa el Banco Mundial como organización internacional ${ }^{6}$ experimenta un cambio que le conduce a la reevaluación de su estrategia y de su relación a uno de sus objetivos fundacionales, el de la reducción de la pobreza mundial ${ }^{7}$. La lucha contra la pobreza o fight against povert $y^{8}$ ganara terreno en la década de los noventa para finalmente posicionarse en la agenda internacional con la llegada del nuevo milenio, marcando una ruptura con el enfoque economicista del desarrollo. Hoy en día, la LCP ha logrado integrar semántica e ideológicamente una multiplicidad de actores y acciones como programas de gobierno, iniciativas de la sociedad civil, esloganes de ONG o titulares de hasta medios de comunicación.

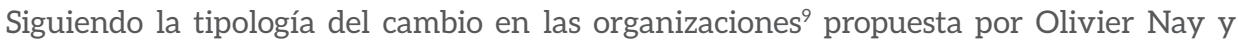
Petiteville, con la adopción de la LCP opera en el BM un cambio en la esfera cognitiva y normativa de la institución. Es decir "un cambio que toca las formas del saber y los conocimientos compartidos por quienes integran la institución (...) así como las representaciones interiorizadas por los actores" (Nay, Petiteville, 2011). La hipótesis que se propone es que efectivamente con el paradigma de la LCP se produce un cambio en la esfera normativa del BM sobre lo que debe ser atacado y como debe ser atacado.

6 Una organización internacional es definida por March y Olsen como un conjunto estable de prácticas y reglas que contribuye a la formación de órdenes políticos internacionales (1998).

$7 \mathrm{El} \mathrm{BM}$ se encuentra en el cruce de sus tres objetivos fundacionales, como institución financiera, debe abogar por la rentabilidad financiera de los préstamos que otorga; como agencia de desarrollo, debe disminuir la pobreza mundial y como centro de investigación, genera marcos de referencia que permiten desarrollar los dos objetivos precedentes. Los dos primeros objetivos de alguna forma en contradicción, y el tercero al servicio de estos.

8 Término popularizado en la administración de Lyndon Johnson 1964, en el cual fijaba como prioridad nacional la erradicación de la pobreza en los Estados Unidos, a través de este se instaura un paquete de medidas sociales, como por ejemplo el Social Security Act de 1965.

9 La tipología incluye cambios de diferente orden: 1. Reformas institucionales, 2. Reconfiguraciones de poder en el seno de la organización y 3. Cognitivo y normativo. 
Lo anterior se tradujo en una diversificación de los tipos de intervención financiables y en la producción y difusión de un saber-hacer relacionado. Más que un imperativo moral, la LCP delimita una forma de entender la pobreza, un tipo de intervención financiable, unos sujetos de intervención y unas condicionalidades que deben cumplirse.

Para contextualizar la importancia de este cambio, es necesario realizar un breve recorrido sobre el accionar del BM. En los años sesenta, una vez la Europa de postguerra reconstruida -primera función del BM- y las colonias en proceso de independización, el accionar del BM se orientará a la capitalización de las economías menos desarrolladas. Para esto, será determinante la creación de la Agencia Internacional para el Desarrollo (AID) en 1960, la cual otorgará préstamos a tasas favorables a los PED en los años sesenta y setenta. En este periodo el banco promueve una perspectiva liberal del desarrollo, por lo cual su intervención se basó principalmente en obras de infraestructura y en los sectores considerados productivos.

El siguiente periodo (finales años setentas y ochentas) estará caracterizado por la dificultad de los PED para responder a las obligaciones contraídas con la institución, para lo cual es determinante el contexto de crisis económica de los años setenta (crisis petrolera, aumento de materias primas, aumento de tasa interés E.E.U.U).

Los planes de ajuste estructural (PAE) surgen en este contexto, donde el BM - de la mano del FMI- se consagra como líder mundial de las políticas de desarrollo. Para esto, será determinante la influencia intelectual de la investigación que se desarrollaba en su seno (fortalecida en la presidencia McNamara 1968-1981), la cual fundamenta una serie de intervenciones de corte neoliberal que más tarde serán conocidas como el Consenso de Washington. Los países que contraen estos planes serán intervenidos en su política económica y sectorial, al tiempo que se pondrán en marcha los procesos de liberalización económica y de privatización en un periodo que va hasta los años noventa (Devin et Placidi, 2011).

Los PAE tendrán un impacto contrastado en los países que los contraen, en general, se observa una degradación de los indicadores de desarrollo y un aumento en la incidencia de la pobreza, en particular para el caso africano. Retrasando así, el proceso de movilidad social que en algunos países se estaba gestando, generando consecuencias antidistributivas (Pastor, 1987).

Un primer antecedente sobre la relación entre LCP y PTC, la encontramos en el simposio "poverty and ajustment" realizado en 1988 por el BM, el cual será luego condensado en el documento "targeted programs for the poor during structural adjustment". En este se discute el impacto de los PAE para los pobres y surgen los programas focalizados como opciones que les permitirían atenuar la emergencia. Los programas focalizados se presentan como intervenciones costo-eficientes (consideradas las intervenciones menos costosas en términos presupuestales y políticos) y se conciben como medidas compensatorias y temporales. Todo esto, teniendo como trasfondo la idea de no desincentivar la inserción laboral con transferencias cercanas al nivel del salario mínimo y el rol de la inversión en capital humano para la superación de la pobreza. 
Esta reconsideración del tipo de intervención del BM surge dentro de la crisis de legitimidad que golpea la institución, que la obliga a replantear su accionar hacia los PED. La década de los noventa es reveladora de esta transformación y el antecedente será el reporte de desarrollo mundial de 1990, donde la LCP se eleva a título de imperativo moral y se fija como objetivo, la reducción a la mitad de la pobreza extrema (monetaria) para 1995, objetivo que será luego retomado por el PNUD con la firma de los ODM en el año 2000.

Por su lado, la publicación de los reportes de desarrollo humano del PNUD desde 1990, contribuye a la tematización de la pobreza en la agenda de los PED. Esta organización innovará en el campo, al integrar concepciones no-monetaristas de la pobreza como el índice de desarrollo humano (1991) y del índice de pobreza humana (1997), concebidos por los economistas Mahbub Ul Aq y Amartya Sen. Por ultimo cabe destacar que en la cumbre social sobre desarrollo social de la ONU realizada en Copenhague en 1995, se declara la necesidad urgente de resolver el problema de la pobreza en los PED.

Volviendo al BM, los documentos estratégicos de lucha contra la pobreza serán publicados a partir del año 1999, reuniendo líneas directrices y recomendaciones para la reducción de la pobreza en cada país. Con esto, la institución pretendía reconocer la especificidad de cada contexto, lo cual significaba romper con la estrategia de los años sesenta y setenta.

Pero será el reporte sobre desarrollo en el mundo del año 2000 titulado "attacking poverty", donde el BM fijará el cuadro de acciones de la LCP. En este se introduce un conjunto de medidas que involucran el manejo del riesgo, la importancia de la construcción de instituciones sociales por parte del Estado, la idea de focalización y conceptos como el de empoderamiento y privación humana (inspirados de los aportes de Sen). Además, en la década de los 2000 surgen nuevos instrumentos de financiación conocidos como "Poverty reduction strategy credits".

En América Latina esta lucha será acogida por el BID y la CEPAL pero también por las organizaciones multilaterales regionales. En la Cumbre de las Américas realizada en Chile en el año 1998, la LCP será definida como prioridad para los gobiernos latinoamericanos. Fecha que coincide con las primeras experimentaciones de PTC en México y Brasil, los cuales se difundirán y adaptarán a los contextos nacionales en un escenario que los acoge y se beneficia de la tutela de los expertos (Debonnevile, Diaz, 2011).

Por su lado, la firma de los objetivos del milenio (ODM) en el año 2000 -en particular su primer objetivo de reducir la pobreza monetaria a la mitad para 2015- va a dotar de un carácter de urgencia a la LCP.

A modo conclusión de la sección, la tematización de la LCP constituye un paradigma que si bien no transforma la naturaleza del BM, sí reorienta su accionar y el enfoque de las investigaciones. La LCP permite a esta institución ratificar su influencia en la definición e implementación de políticas públicas de desarrollo en los PED. Sin embargo, el éxito de esta estrategia no habría sido posible sin la configuración de diferentes organizaciones internacionales y del sector solidario, ni tampoco sin la inyección de recursos que las agencias del BM tendrán luego de la crisis del 2008. 


\section{FUNDAMENTOS TEÓRICOS DE LOS PTC.}

Los PTC como instrumentos no se constituyen independientemente de una producción de conocimiento especializado, de hecho estos son portadores de un saber-hacer en el tratamiento de la pobreza que se alimenta de diversas teorías que se enmarcan dentro de la rama de la economía del desarrollo. Los PTC combinan la concepción de la pobreza de Amartya Sen, el concepto del capital humano aplicado al desarrollo, la doctrina del manejo social del riesgo y por último, la idea de focalización de la asistencia. Componentes que constituyen las tecnologías de intervención que Sonia Álvarez denominaría partes constitutivas de la nueva economía política de la pobreza (Álvarez, 2013).

En el caso de los PTC, la innovación consistió en plantear un mecanismo de condicionalidad, mediante el cual se otorga una transferencia monetaria (en efectivo) a una familia a cambio de la escolarización y del cumplimiento de controles de salud de niños y adolescentes.

Es importante entender las transferencias condicionadas como subsidios a la demanda que buscan la reducción de la pobreza en dos series de tiempo. En el corto plazo se busca aliviar la pobreza monetaria con un complemento al ingreso del hogar que a su vez impacta el consumo global. En el largo plazo, se busca romper con la transmisión intergeneracional de la pobreza gracias a la condicionalidad sobre el capital humano, la cual permite un aumento en los niveles de escolaridad y de nutrición, lo cual se asume aumentará el ingreso futuro de los niños, permitiendo la superación de las trampas de pobreza $^{10}$. A continuación se describen los conceptos subyacentes que influencian la construcción conceptual de los PTC.

\subsection{El Manejo Social del Riesgo (MSR).}

La doctrina del MSR surge como un nuevo enfoque de protección social que se adapta a los desafíos del mundo globalizado e integra la agenda global del desarrollo. Este enfoque será desarrollado a finales de los noventa y comienzo de los años 2000 bajo el liderazgo del economista Robert Holzmann quien encabezaba la oficina de protección social y del trabajo del BM. El BM incluye este concepto desde el informe de desarrollo del año 2000.

La doctrina del MSR nos ofrece una lectura de la protección social basada en el concepto de riesgos (entendido como elementos que amenazan el ingreso de los hogares) y donde la pobreza es vista como un evento transitorio ligado a un riesgo que no se supo prevenir en su momento (Holzman et Jogerzenn, 2000).

Se busca entonces reducir el nivel de gasto público trasladando gran parte del manejo del riesgo al sector privado y en particular al sector asegurador para los agentes que pueden costearlo. A su vez se plantean modelos de responsabilidad compartida entre varios actores para manejar el riesgo asociado a los pobres. El rol del Estado es entonces

$10 \mathrm{El}$ concepto se introduce con los trabajo de Jeffrey Sachs (2005) el cual ha sido posteriormente trabajado por Esther Duflo (Banerjee et Duflo, 2012). Se producirá una trampa de la pobreza cada vez que el crecimiento de la renta esté limitado para quienes tienen poco para invertir, mientras que crece rápidamente para quienes puedan invertir un poco más. De esta forma, los ingresos futuros son inferiores a los ingresos presentes para quien se encuentra en esta lógica. 
el de hacerse cargo de los riesgos que le resulta muy costoso proveer al mercado y que sobrepasen la capacidad de los mecanismos informales de protección.

El MSR asociado a la regulación de la pobreza entra como competencia estatal pero también involucra al sector privado (bancarización y acceso al sector financiero de los pobres) al sector solidario (ONG) y a los mecanismos informales de protección como activos de los pobres (los cuales entran como capital y son movilizados solo en la medida que no vayan en contravía del desarrollo económico).

Dentro de las medidas concebidas para disminuir los riesgos a los que están expuestos los pobres se encuentra las acciones de prevención, mitigación y recuperación. Por un lado, se busca proteger a los hogares de los riesgos que puedan afectar sus medios de subsistencia y por el otro, se busca incentivarlos a participar en sectores más riesgosos pero más rentables.

La asistencia social enmarcada dentro del sistema de protección social entra como medida de recuperación, y en tanto que integrantes de este eje, los PTC. A pesar de esto, la experiencia latinoamericana demuestra que las transferencias dejaron de ser medidas de recuperación temporales para ser permanentes.

Para concluir, es importante señalar que el MSR contiene una forma de pensar los riesgos y los momentos no productivos de la vida de un individuo. La crítica que este suscita, es que el MSR plantea una política social basada en el manejo de los activos de los individuos y no en los derechos (Lampis, 2011). Por lo cual, el individuo antes de afirmarse como ciudadano lo hace como cliente, de alguna forma la protección social se piensa en términos de riesgo, pero el riesgo principal que se busca evitar es aquel relacionado con la pérdida del ingreso futuro. Para Moser (2001) esta visión del riesgo ligada al ingreso nos devuelve a una visión monetarista de la pobreza. Su crítica se basa en las categorías que este modelo utiliza indistintamente para varios riesgos, lo que no permite encuadrar intervenciones que por naturaleza son transversales. Por otro lado, Moser resalta que el modelo del MSR será extrapolado del contexto africano, donde se presentan otro tipo de riesgos y donde los sistemas de protección social se encontraban en otra etapa al comienzo del siglo XXI.

Por último, pensar la protección social en términos individuales implica desconocer el principio de solidaridad como principio constitutivo de los sistemas de protección social, lo cual es fuente de vínculo social en una sociedad (Paugam, 2008), lo que a su vez genera corresponsabilidades con el Estado y sentido de pertenencia en los ciudadanos.

\subsection{La teoría de las capacidades de Amartya Sen.}

Varios conceptos trabajados por el economista indio Amartya Sen serán tenidos en cuenta dentro el componente teórico-conceptual de los PTC. En la década de los noventa, Sen elabora una crítica a la teoría neoclásica ${ }^{11}$ y su manera de considerar el bienestar. Para este autor, la pobreza debía ser evaluada no como la dificultad de acceso a ciertos bienes y servicios (Rawls) ni en términos de la utilidad (placer y deseos), sino tomando como referente un 
conjunto de capacidades que permitan a los individuos ejercer su libertad. El conjunto de capacidades permitirían a los individuos transformar sus recursos en funcionamientos (lo que efectivamente realizan los individuos). Se trata de una teoría que vincula la pobreza con la falta de libertad. Con esto, Sen buscaba reconocer las diferencias y particularidades ligadas a los diferentes contextos socioculturales, al mismo tiempo que trasladar el debate sobre los recursos con los que cuentan las personas, a lo que estas puedan efectivamente hacer con estos.

La teoría de Sen ha tenido una amplia recepción y es gracias a sus aportes que las definiciones monetarias de la pobreza cederán ante una definición en términos de las privaciones o carencias de los individuos. Bajo esta influencia teórica, el PNUD ${ }^{12}$ optará por la integración de medidas multidimensionales de la pobreza como el índice de desarrollo humano (aparecen como dimensiones longevidad, nivel educativo y nivel de vida decente) y más tarde, por el índice multidimensional de la pobreza que define la pobreza en términos de calidad de vida.

Ahora bien, las modalidades de los PTC se verán influenciados por esta teoría en varios aspectos. Primero, los PTC son entendidos como programas que desarrollan capacidades, principalmente al buscar aumentar las capacidades futuras de los individuos gracias a la condicionalidad impuesta sobre el capital humano. Segundo, el libre uso de las transferencias se inspira de la voz y la libertad que Sen quiere devolver a los pobres. Tercero, los PTC fueron concebidos para ser administrados por las mujeres, lo cual se presenta desde esta teoría como herramienta para aumentar el poder de decisión y agencia que estas poseen en el plano doméstico.

En su ensayo "La economía es una ciencia moral", Sen considera las políticas destinadas a complementar el ingreso como una manera eficaz de prevenir las hambrunas, lo que permite reconstituir el ingreso de los más pobres y estabilizar su poder adquisitivo en momentos de crisis (Sen, 1998, P. 53).

Ahora bien, la teoría de las capacidades ofrece un amplio margen de aplicación, pero también de instrumentalización. La crítica que se realiza parte de la misma teoría que propone Sen, de hecho, el autor ha formulado una definición mucho más amplia de su teoría de las capacidades, la cual nos permite realizar una crítica del enfoque que propone el PTC para el caso colombiano.

Se trata de la inclusión de los factores de conversión como determinantes para la conversión de los recursos en capacidades y posteriormente en funcionamientos. En esta teoría los recursos que poseen los individuos pueden ser convertidos en capacidades solo sí lo permiten los factores de conversión. Se trata de tres tipos de factores que Sen describe. Primero, los factores individuales comprenden las características, competencias y habilidades propias de los individuos. Segundo, los factores sociales incluyen el contexto sociopolítico y cultural, lo que incluye elementos sociales como las normas, los tabúes, la relación de género, la estabilidad política o la discriminación. Finalmente, los

\footnotetext{
12 Aunque con una mayor reticencia, el BM involucrará progresivamente los conceptos de Sen en sus informes, principalmente en lo que se refiere al concepto de empoderamiento o empowerment (dar voz a los pobres), el cual se define también en términos de expansión de la libertad.
} 
factores ambientales incluyen la infraestructura y el entorno necesario para la adecuada utilización de los recursos (Sen, 1999). Esta parte de la teoría reconcilia el individualismo metodológico con una visión holista que tiene en cuenta la complejidad de los hechos sociales. Lastimosamente, más allá de un llamado a la necesidad de la democracia o de contextos políticos estables, esta parte de la teoría será poco explorada y operacionalizada por las instituciones y teóricos.

Los PTC adoptan la teoría de Sen, haciendo caso omiso del fortalecimiento e importancia de los factores sociales y ambientales que condicionan la situación de pobreza. Los PTC al hacer énfasis en los factores individuales (formación en capital humano), no ofrecen el marco para que los recursos se traduzcan en libertades reales, de manera que los derechos permanecen formales y no reales (Bonvin, Farvaque, 2007). Si nos ceñimos al modelo planteado por Sen, se podría decir que mientras estos factores sociales y ambientales no sean asegurados, cualquier intervención que busque aumentar los recursos o las capacidades de los pobres no lograría el impacto estimado.

Como última crítica a la adopción del enfoque, el individualismo metodológico del que Sen parte contribuye a hacer de la superación de la pobreza una cuestión individual o familiar. Uno de los efectos prácticos de esta concepción observados en terreno, es que la superación de la pobreza es vista como un asunto que depende de las habilidades y decisiones de los jefes de hogar, lo que contribuye a la culpabilización de los beneficiarios y a una movilidad social individual y no grupal (Ayola, 2016).

\subsection{Condicionalidad.}

Otra de las innovaciones de los PTC es que estos logran alejarse de las políticas tipo Workfare (condicionar la recepción de ayudas sociales a la prueba de inserción laboral) como forma de prevenir la aparición de la figura del "free-rider", una de las principales preocupaciones de la economía neoliberal.

De hecho, las transferencias al ser de libre destinación, permiten a los beneficiarios disponer libremente del dinero que reciben sin tener que comprobar esfuerzos para la inserción laboral. De acuerdo a Cecchini y Madarriaga "Los programas utilizan principalmente transferencias monetarias de libre uso ya que se considera que los propios hogares son los que mejor uso pueden hacer de dichas transferencias sobre la base de sus preferencias" (Cecchini y Madarriaga, 2009). De alguna manera se encuentra subyacente la racionalidad del consumidor -como aquella que sustenta la teoría de las preferencias de Samuelson-, pero también se exalta la libertad como elemento que permite el empoderamiento, concepto que se encuentra en la teoría de Sen.

La condicionalidad entonces no fue planteada en torno al uso de las transferencias recibidas, a pesar de esto, los montos de las transferencias fueron planteados lo suficientemente bajos ${ }^{13}$ para no crear incentivos perversos que impactaran la inserción

13 En Colombia estos también fueron teniendo en cuenta las tasas de deserción escolar, es decir que para los cursos donde existe menor abandono como en primaria, bajo la lógica del incentivo las transferencias, se otorga un subsidio de menor cuantía. Hoy en día el subsidio para los niños en educación primaria es de 15.000 pesos cada dos meses. 
laboral. Por el contrario, la condicionalidad fue planteada en términos de capital humano, donde se parte de un prejuicio tutelar que los pobres no aplican la racionalidad económica a la hora de realizar la decisión de cuidar o educar a sus hijos.

La rama de la economía del desarrollo que se sustenta en experiencias empíricas, ha profundizado en los impactos económicos del comportamiento de los pobres. Para Banerjee y Duflo (2012) las decisiones erradas que los pobres toman -como por ejemplo abandonar la escuela o no sanarse- se explican no solo por sus limitadas dotaciones de base sino por comportamientos como la procastinación, un elevado sentido de lo inmediato así como una subestimación de los beneficios reales. Por esta razón la incitación resulta el método más adecuado para lograr los objetivos de reducción de la pobreza (Banerjee et Duflo, 2012, P. 70, 93). La condicionalidad busca así convertir en un cálculo costo-beneficio la inversión en capital humano para las próximas generaciones.

\subsection{Capital humano.}

El capital humano (KH) es desde los trabajos de Becker (1964) considerado un tipo de capital susceptible de rendimientos futuros. Pero será con los trabajos de Romer y Barro (1990; 1991) que la inversión en capital humano se integra a la función de producción y, en esta misma línea, se establece una correlación entre esta dotación y el crecimiento económico.

Teniendo como referente el concepto de trampas de pobreza, la economía del desarrollo ha buscado aumentar el stock en $\mathrm{KH}$ de los pobres para que de esta forma las generaciones futuras tengan mejores ingresos y logren ser más productivas, lo que se espera, les permitirá la superación de la pobreza. Así, el enfoque desde el capital humano, nos habla de un capital (educación, salud) que es productivo y productor.

Con los PTC se invierte en educación atacando la deserción escolar. Por un lado, se asegura la demanda del servicio y aumentan los años de escolaridad de los infantes. Mientras que por otro lado, se previene la posibilidad de que los padres ante choques externos (desempleo, crisis) tomen decisiones contraproducentes, como sacar los niños de la escuela, que afecten la productividad futura del hogar.

La crítica que subyace es que desde la óptica de los PTC, el KH está siendo considerado principalmente desde el componente de la demanda del servicio público (asistencia escolar) y no desde la oferta del servicio (infraestructura, cobertura, calidad, pertinencia). Adicionalmente, la asistencia escolar no es garantía de logro educativo como lo han mostrado las evaluaciones de impacto realizadas al programa en Colombia (Benson, 2012).

4.5. Focalización. Los PTC encierran una tecnología de la focalización, la cual tiene como principal objetivo la búsqueda de la eficiencia en las intervenciones sociales en un escenario de escasez de recursos. La teoría neoliberal contempla la intervención estatal solo en la medida que esta no genere distorsiones el mercado. Por lo tanto, focalizar se convierte en la manera para concentrar los recursos allí donde estos serán más eficientes, es decir en los individuos que tienen las menores dotaciones de base: los más pobres de los pobres. 
Es por lo anterior que ha resultado necesario el desarrollo de tecnologías e instrumentos de focalización que permitan que las tareas de selección y asignación se realicen con información centralizada y fiable. Actualmente cada Estado que ha adoptado los PTC maneja sistemas de identificación diferentes, lo que va desde la simple encuesta hasta la sistematización compartida. Todo esto, como forma de garantizar la eficiencia en la intervención y efectos de inclusión de "free-riders" o polizontes.

La discusión sobre la pertinencia de la focalización en Colombia es la existencia de una gran cantidad de personas inscritas en el Sisben que no acceden a la prestación. Focalizar no solo pone en cuestión el principio de igualdad (Art. 13 C.P) sino que genera una categorización de la sociedad entre los pobres -objetos de la solidaridad internacional- y los no pobres, que deben acceder a través del mercado a los derechos sociales.

\section{EL PTC FAMILIAS EN ACCIÓN: UNA MIRADA CRÍTICA AL CONSENSO A SU FAVOR A LA LUZ DE ALGUNOS RESULTADOS E INDICADORES.}

Los PTC entran en América Latina como respuesta a situaciones particulares, generalmente de crisis ${ }^{14}$, que atravesaron los países de la región a finales de los noventa y a principios de los años 2000. En el caso colombiano, el programa piloto de Familias en Acción se implementa en la presidencia de Andrés Pastrana (1998-2001) en el año 2001, como parte de la Red de Asistencia Social (RAS) del Plan Colombia (2000). El programa cumple entonces dieciséis años en funcionamiento y cinco años desde su inscripción como política de Estado.

Desde su implementación y las evaluaciones que le seguirán se afirma que los PTC se enfocann al origen de la reducción de la pobreza experimentada en los últimos quince años en América Latina. Es un hecho que desde los años noventa la pobreza monetaria ha logrado reducirse en la región y el caso colombiano confirma esta tendencia (Cepal, 2015). Entre 1991 y 2001, la incidencia de la pobreza aumenta en 3,3 pp, mientras que para el periodo 2002-2016, esta se reduce en 21,7 pp (DANE, 2016). Es importante mencionar que esta metodología sufre un cambio en el año $2011^{15}$.

En este punto, es interesante anotar que para el año 2016 la incidencia de la pobreza aumentó por primera vez en más de quince años. La incidencia de la pobreza aumentó en 0,2 pp, lo que la ubicó en un 28\%, mientras que la pobreza extrema aumentó en 0,6 pp, llegando a 8,5\% de la población. Ambas tendencias se registraron en particular para los centros urbanos.

Ahora bien, el DANE emplea una medida desde el año 2013, denominada descomposición de la pobreza, la cual se basa en los trabajos de Shorrocks y Kolenikov (2003). Esta metodología evalúa el impacto de tres componentes en la variación de la incidencia de pobreza: el cambio en la línea de pobreza de un año a otro (ajuste por inflación de la línea), el crecimiento del ingreso y la redistribución.

14 Para la Argentina será la crisis económica del 2001, para Brasil será una negociación entre el gobierno del expresidente Lula y el BM, para Chile serán los efectos de la crisis asiática.

15 La Mesep Misión para el Empalme de las Series de Empleo, Pobreza y Desigualdad fue creada en enero de 2009 mediante un convenio DANE-DNP. En su segunda fase de trabajo, la Mesep diseña una nueva metodología de medición de pobreza monetaria, la cual se aplica desde el año 2009. 
Según esta medida para el periodo 2012-2013, teniendo en cuenta que el crecimiento económico fue de 4,3\% para 2013 y que la incidencia de la pobreza se redujo en 2,1 puntos porcentuales (pp), la disminución de la LP nacional fue efecto del aumento del ingreso (-2,9). En el periodo 2013-2014, cuando el crecimiento económico fue de 4,6\% y la reducción de la incidencia de la pobreza fue de 2,1 pp, la medida nos indica una tendencia parecida a la del año anterior, en efecto, la reducción fue producto de un aumento del ingreso de 2,9 pp. Para el período 2015-2014 la situación se perfila diferente, teniendo en cuenta que la reducción de la pobreza fue menor $(-0,7$ pp) pero también el crecimiento económico (3\%), se encuentra un efecto más importante de la redistribución (-1,9) que en el crecimiento económico $(-0,8)$ a la hora de reducir la incidencia de la pobreza. Ahora bien, para el año 2016, cuando el crecimiento fue de $2 \%$, el factor que incide en mayor medida es el cambio en la línea de la pobreza o ajuste por inflación, el cual influye en un aumento de 3,3 pp de la pobreza.

Un análisis detallado de esta medida es necesario para entender la dinámica de la reducción de la pobreza en Colombia, sin embargo, los datos revelan que el efecto del crecimiento económico ha sido mucho más importante que el efecto de la redistribución. El cambio que se observa en los dos últimos años, en un periodo de desaceleración económica, nos indica que los hogares pobres no logran de un año a otro recobrar el valor adquisitivo de su ingreso, es decir que sus ingresos se mantengan de un año a otro.

Por otro lado, el hecho que la redistribución no logre jalonar las cifras de pobreza, nos habla de una insuficiencia de las estrategias de política social y de su limitado impacto para mitigar los efectos de la desaceleración económica. Esto, sin poner directamente en duda el PFA, sí se vuelve un antecedente para cuestionar el rol de la política de asistencia social como ese colchón que mitigue el riesgo de los efectos de la crisis y de la dinámica macroeconómica no favorable.

El paradigma de la LCP se construye alrededor de la reducción de la pobreza, la cual confirman en Colombia las cifras privilegiadas para su medición, sin embargo, partiendo del concepto de la política social como herramienta redistributiva, no se puede omitir la pregunta por la desigualdad y el efecto que sobre esta tiene el PTC Familias en acción. El análisis sobre el tipo de movilidad social que han experimentado aquellos que han logrado superar la pobreza ha sido postergado por las evaluaciones de impacto.

Dos medidas se acercan a esta pregunta sobre la desigualdad de ingresos. El coeficiente de Gini y los datos que nos proporciona un reciente reporte del BID. Primero, el coeficiente Gini desde finales de la década de los noventa se mantiene por encima de 0,5. En efecto, Colombia entre el periodo 2011-2014 sólo vera el Índice de Gini reducirse en un 0,004. Los resultados del 2015 afirman una reducción de la desigualdad con un Gini de 0,522 ${ }^{16}$, la cual continúa para 2016 donde se observó un coeficiente de 0,517.

En esta misma línea, la desigualdad medida por fuentes de ingreso tributarios que nos propone la Cepal. Para 2014 Colombia respecto a América Latina fue el país con la mayor 
participación del 1\% más rico en el ingreso total a lo largo de toda la serie 1993-2014, para 2010 este segmento capturaba el 20,5\% del ingreso total del país (Cepal, 2015).

Un reciente reporte del BID, muestra una radiografía interesante de la estructura de ingresos colombiana que nos permite visualizar la pirámide económica (BID, 2015). En el reporte, se clasifica la población de acuerdo a una estimación monetaria del ingreso diario en cinco situaciones del ingreso, las cuales define en términos de clases sociales y población vulnerable -categorías bastante debatibles-. No obstante lo anterior, según este reporte para 2015 la pobreza extrema afectaba a 18,6\% de la población (ingreso diario per cápita menor a US\$2,5), la pobreza moderada un 15,4\% (entre US\$2,5 y US\$4), la población vulnerable representa a un $36,7 \%$ de colombianos (entre US\$4 y US\$10). La clase media abarca a $27,2 \%$ (entre US $\$ 10$ y US $\$ 50$ diario) y por último la clase alta representa $2,2 \%$ de la población (US\$50 o más). De acuerdo a esto, 68,7\% de la población colombiana vive con menos de US $\$ 10$ dólares diarios. Lo que se deduce es que si bien los anteriores pobres han logrado superar el umbral de la pobreza, estos no lo han hecho para lograr una estabilidad propia de la clase media.

El gasto social es sin duda el componente más importante a través del cual el Estado incide sobre la distribución del ingreso. Sin embargo, se observa una tendencia a la disminución del gasto social destinado al programa en los últimos dos años. Para 2009 la inversión en FA representaba 0,39\% del PIB, lo que constituía 3,14\% del total de la inversión social (Cepal, 2011). Para 2015, esto se reduce a 0,27\% del PIB y para el año 2016, la Cepal alerta de una reducción de 276 millones de dólares para el programa (Cecchini y Atuesta, 2017). El pronóstico es que el ciclo macroeconómico bajista que se espera para Colombia tendrá repercusiones en la inversión de los PTC, así, de acuerdo a Clavijo con la contracción real del 3\% sobre el presupuesto general de 2018, la asignación para Familias en Acción descenderá a un 0,1\% del PIB (Clavijo, 2017).

Otro argumento que permite postular su eficiencia es la tasa de cobertura que el programa permite a bajo costo. FA es el programa de asistencia social de mayor cobertura del país, este se presenta como "una de las operaciones con mayor cobertura y escala presupuestal en la política social colombiana" (Angulo, 2016). De hecho, aumentó su cobertura casi nueve veces desde 2002 a 2014, al pasar de 320 mil familias a 2,67 millones de familias cobijadas, aun cuando para 2016, la cobertura disminuye a 2,5 millones de hogares. Colombia figura como uno de los países con mayor nivel absoluto de cobertura de hogares en la región ${ }^{17}$, junto aArgentina, Brasil, México y Perú (Cecchini y Atuesta, 2017). Efectivamente el programa cumple con una cobertura que ningún otro programa social ha logrado.

Para 2015, el porcentaje de personas que vive en hogares destinatarios fue de $21,7 \%$, ubicándose cerca del promedio para América Latina de 20,9\%. Los PTC se perfilan como costo-eficientes porque a bajo costo permiten tasas de coberturas tan importantes como estas.

No obstante, el programa aún experimenta efectos de exclusión, los cuales pueden llegar

17 Este programa ha experimentado varias etapas que podemos clasificar en tres periodos, 2002-2007, 2007-2012, 2012-2016. Entre el periodo 2005 y 2013 la tasa de progresión de la cobertura fue de $+386 \%$, lo cual comparado con Brasil (+51\%), Chile (+100\%) o México (+32\%) lo ubica como uno de los programas que más aumentará su cobertura familiar en términos relativos en América Latina (Collombet, Leprince, 2015). 
a ser más importantes que los errores de inclusión de acuerdo a Cechinni y Madarriaga (2011). El programa desde su concepción excluye de la transferencia personas que aun cuando puedan encontrarse bajo la línea de pobreza, no cumplen con requisitos tales como no tener un hogar con hijos o contar con un domicilio fijo. Además es importante recordar que son 36.7 millones de colombianos (75,3\% de la población) los inscritos en el Sisben, dentro de los cuales un 63.5\% es considerado en condición de alta vulnerabilidad y 30 millones clasificados en Sisben I (Clavijo, 2017).

Las altas tasas de cobertura del programa se logran con unos relativos bajos costos, pues a diferencia de la inversión en bienes y servicios sociales, las transferencias no necesitan altos costos de funcionamiento ni inversión en infraestructura. Sin embargo, para el caso colombiano la cobertura del programa se logra a expensas de un monto reducido de la transferencia. De hecho, la inversión anual per cápita en hogares destinatarios en Colombia es mucho menor que la de otros países de la región. Efectivamente, el país figura entre los seis últimos de la región con una inversión de \$69 dólares por persona anuales, lo cual contrasta con el promedio regional que es de \$153US (Cecchini y Atuesta, 2017).

Asimismo es importante señalar como determinante del costo presente y futuro del programa el tipo de financiación que este tuvo en las dos primeras etapas, es decir el de crédito externo. Desde su implementación hasta el año 2010 el programa habría costado US $\$ 3070$ millones, de los cuales 54,1\% fueron financiados vía crédito externo (31,8\% BM y $22,3 \%$ BID) y $45,9 \%$ por la nación, de los cuales $11,1 \%$ eran recursos del ICBF (Presidencia y DNP, 2010). El bajo costo del programa se podría ver relativizado si se realizan las proyecciones que permitan entender el impacto del programa en el servicio a la deuda colombiana en el mediano y largo plazo.

Ahora bien, desde que estos programas se ponen en marcha han sido arduamente evaluados y monitoreados por las instituciones financiadoras, convirtiéndose en un objeto privilegiado de la producción científica. Ha sido evaluado principalmente en la relación del programa con los efectos de la condicionalidad, es decir la inversión en capital humano, por lo general con técnicas experimentales entre grupos de control y de tratamiento.

Algunos de los resultados en el componente salud y nutrición son: 1. En un año de participación en el programa FA disminuye un 1\% la prevalencia de desnutrición crónica (Attanasio, Trías y Vera-Hernández, 2009).2. El consumo de proteínas aumenta en forma más proporcional que el de alimentos en general (Attanasio, Mesnard, 2005). 3. Se observó un aumento de estatura de 1,3 cm para los niños y niñas de 9 años y un incremento de 1,5 cm para los niños y niñas de 12 años (Attanasio 2012).

En cuanto a educación, se ha encontrado que los niños que reciben el subsidio son en un 4 a 8 por ciento más proclives a terminar sus estudios. Al examinar los impactos de logro escolar en el largo plazo, se encuentra que el PTC colombiano no incide en una mejora del desempeño escolar, en efecto, quienes asisten a la escuela incentivados por la transferencia, en su desempeño no presentan diferencias significativas respecto a los no beneficiarios (Báez, Camacho, 2011), los resultados en el logro escolar no son contundentes (Benson, 2012), de acuerdo a algunos resultados el programa parece incluso estar 
relacionado negativamente el desempeño escolar de los beneficiarios en zonas rurales (García, Hill, 2009).

Lo anterior entonces permite entender que si bien existen impactos sobre los componentes de salud y educación los cuales son importantes para las próximas generaciones, estos se ven limitados en su alcance. Esto nos permite cuestionar el consenso que existe sobre la eficiencia de estos programas, ya que este tiende a ser desproporcionado con respecto a los logros que se han encontrado.

Finalmente, todo lo anterior invita a mirar con mayor precaución los resultados del programa y en particular el argumento que lo posiciona como el tipo de intervención más eficiente en términos de su costo. Asimismo se busca tener en cuenta que el impacto del programa sobre la reducción de la pobreza, está asociado a otros elementos como el crecimiento económico experimentado en la última década.

\section{CONCLUSIÓN}

En este documento se ha emprendido un análisis de los PTC desde la dimensión global de la LCP que permite su puesta en marcha, difusión y financiación en la primera década del siglo XXI. Siguiendo a Cling, la difusión de la internacional LCP es prueba de la capacidad de resiliencia del BM ante una crisis de legitimidad como la afrontada en los años noventa (Cling et al, 2011). Esta transformación le permite preservar el liderazgo en la definición de las políticas de desarrollo para los PED. Si bien se da un cambio normativo en la institución, el discurso de la institución se trasforma sin dejar de lado una visión de desarrollo orientada a la esfera del mercado y sin abandonar fórmulas de la ortodoxia económica.

Los PTC son actualmente la estrategia privilegiada de LCP en Latinoamérica y su adopción representa la adhesión de los Estados-nación a un nuevo orden. Hacer una lectura de estos como parte de una estrategia global nos permite entenderlos como parte de una reestructuración de la base ideológica de las políticas sociales, donde políticas asistenciales minimalistas se imponen a políticas universalistas basadas en la noción de ciudadanía. Con la LCP, la pregunta por la desigualdad social en América Latina cedió sin aparente respuesta.

La estrategia de focalizar, condicionar e invertir en capital humano, si bien ha contribuido a la reducción de la pobreza en el continente y sus resultados son juzgados satisfactorios, estos siguen siendo parciales en la medida que no se tiene en cuenta el estado de la oferta de servicios de salud y de educación, ni tampoco las oportunidades que ofrece el mercado de trabajo, como determinantes del futuro retorno de la inversión en capital humano.

Además, para el caso colombiano el instrumento no ha superado la prueba de la desaceleración económica, de hecho, la pobreza en Colombia se reduce en la medida que exista un crecimiento económico que lo permita, mientras que el efecto de la redistribución sigue teniendo menor incidencia en la reducción de la pobreza como lo muestra la medida de la descomposición de la pobreza calculada por el DANE. 
En cuanto al instrumento y sus efectos, en el corto plazo este permite a las familias aumentar su consumo (los bajos montos no permiten el ahorro ni la inversión), sin embargo, esto nos devuelve a una visión utilitarista del bienestar, aún muy alejada de una verdadera mejora del conjunto de capacidades de un hogar. En el largo plazo, los resultados son inciertos en un mercado de trabajo que no ofrece oportunidades para los jóvenes menos formados del país que son aquellos que pertenecen a las familias beneficiarias.

Mientras tanto, la focalización cada vez más exhaustiva penaliza a las familias que oscilan la línea de la pobreza, sin lograr una estabilidad propia de la clase media, como lo muestra el informe del BID, ciudadanos que entran a engrosar la categoría de "vulnerables". De esta forma, las medidas redistributivas benefician a una minoría de ciudadanos, mientras que el resto debe recurrir al mercado para procurárselos.

Por último, volviendo al objeto de estudio planteado en la primera sección del documento, el desfase de consideración del programa entre expertos y ciudadanos ordinarios es sintomático de un programa que se implementa de una manera top-down y sin debate público. El cual aplica una fórmula genérica a los países en desarrollo sin tener en cuenta las especificidades ni las causas estructurales de la pobreza estructural ni de la desigualdad social en este contexto.

\section{REFERENCIAS BIBLIOGRÁFICAS}

Agencia Presidencial para la Acción Social y DNP. (2010). El camino recorrido. Diez años Familias en Acción. DNP: Bogotá.

Attanasio, O.; Trías, J.; Vera-Hernández, M. (2009). Old and new welfare: the relative effect on child nutrition. The Initiative of Latin America and the Caribbean without Hunger. Working Paper n. 8 .

Attanasio, Orazio et. al. (2005). How effective are conditional cash transfers? Evidence from Colombia. The Institute for Fiscal Studies: (54).

Ayola-Betancourt, V. (2015), La régulation de la pauvreté en Colombie au prisme de la théorie des liens sociaux: le cas de Familias en Acción. (tesis inedita de maestría). École des hautes Études en Sciences Sociales. Paris.

Ayola-Betancourt, V. (2016). La asistencia social vista por los beneficiarios de un programa de lucha contra la pobreza. Revista OPERA. No. 18. Pp.85-104.

Baez J. et Camacho. A. 2011. Assessing the Long-term Effects of Conditional Cash Transfers on Human Capital: Evidence from Colombia. (N.5681). World Bank policy research working paper: Washington.

Banerjee A, et Duflo E. 2012. Repenser la pauvreté. éditions du Seuil: Paris.

Benson H, A. (2012). Efectos de Familias en Acción sobre la calidad de la oferta educativa. Desarrollo y Sociedad, (70), 51-91.

Bonvin, J-M, et Farvaque, N. (2007). L'accès à l'emploi au prisme des capabilités, enjeux théoriques et méthodologiques. Formation emploi (98), 9-22. 
Cecchini, S. et Atuesta. B. (2017).Programas de transferencias condicionadas en América Latina y el Caribe. CEPAL Serie Políticas Sociales No. 224.CEPAL.

Cechinni et Maldarriaga. 2011. Programas de Transferencias Condicionadas, No.95. Cuadernos de la CEPAL: Santiago de Chile.

CEPAL. 2015; 2016. Panorama social de América Latina. Cepal: Santiago de Chile.

Cling et al. (2011). La banque mondiale, entre transformations et résilience. Critique international. No.53. p.43-59.

DANE. 2013, 2014, 2015. Boletín de prensa Pobreza Monetaria y Multidimensional en Colombia. DANE: Bogotá D.C.

Debonneville et Diaz. (2011). Innovation, diffusion et rôle des idées : une lecture internationale des Conditional Cash Transfers aux Philippines. Congrès 2011 de l'AFS: Ginebra.

Devin, G. et Placidi-Frot, D. (2011). Les évolutions de l'ONU : concurrences et intégration, Critique internationale 2011/4 ( $\mathrm{n}^{\circ}$ 53), p. 21-41.

Holzmann et Jorgensen. (2000). Gestion du Risque sociale: Théorique cadre de la Protection sociale. Discussion Paper Series. no. SP 6. Banco Mundial: Washington, DC.

Kolenikov, S. \& Shorrocks, A.F. (2003). A Decomposition Analysis of Regional Poverty in Russia. Discussion Paper 2003/074. Helsinki: UNU-WIDER.

Lampis, Andrea, (2011). Desafíos conceptuales para la política de protección social frente a la pobreza en Colombia. Revista Estudios Sociales: No. 41, P.107-121.

LAPOP (2012). El barómetro de las Américas: Colombia 2012. Vanderlit University.

Lautier, Bruno. (2013). Les CCTP en Amérique Latine: Entre modélisation internationale et conjonctures politiques nationales. Revue Tiers Monde: N.214, avril-juin.

March J.M, et Olsen, J. (1998), The Institutional Dynamics of International Political Orders, International Organization, 52 (4), 1998, p. 948.

Merrien, François-Xavier. (2013). La protection sociale comme politique de développement : Un nouveau programme d'action international. International Development Policy. P. 68-88.

Olivier Nay, Franck Petiteville (2011). Éléments pour une sociologie du changement dans les organisations internationales, Critique internationale 2011/4 ( $n^{\circ} 53$ ), p. 9-20.

Paugam Serge, Le lien social, collection Que sais-je ?, PUF, Paris, 2008.

Pastor, Manuel (1987). The International Monetary Fund and Latin America: Economic Stabilization and Class Conflict, Westview Press: Boulder.

Pulido, Claudia, (2013) Los programas de transferencias condicionadas en América Latina, el caso de Familias en Acción, Tesis maestría Facultad de Derecho, Ciencias Políticas y Sociales, Universidad Nacional de Colombia: Bogotá.

Sachs, Jeffrey. (2006). The End of Poverty. Penguin Books. 
Sen A. (1996). Freedom, Capabilities and Public Action: A Response, Notizie di Politeia, 12 (43-44), pp. 107-125.

Sen A. (1998). L'économie est une science morale", édit. La découverte.

Sen A. (2010). L'idée de justice. Flammarion: Paris.

Stampini et al. (2015). Pobreza, vulnerabilidad y la clase media en América latina. Documento de Trabajo del BID: Nº 591.

Valencia Enrique. (2008). Conditional Cash Transfers as a social policy in Latin America, an assesment of their contributions and limitations. Annual Review of Sociology, Vol. 3, 2008. Pp.475-497.

\section{Para citaciones:}

Ayola, V. (2018). Condicionar, focalizar e invertir en capital humano: "Familias en Acción" como receta internacional de lucha contra la pobreza para Colombia. Panorama Economico, 26(1), 37-58.

\section{AUTORA}

Valeria Ayola-Betancourt

Doctorante en Sociología de la Escuela de Altos Estudios en Ciencias Sociales (EHESS) en Francia, en cotutela con la Universidad Nacional de Colombia. Cuenta con Maestría en Economía Aplicada de la Université d'Aix-Marseille (Francia) y Estudios Políticos en la Escuela de Altos Estudios en Ciencias Sociales (Francia). Es profesional en Gobierno y Relaciones Internacionales de la Universidad Externado (Colombia). 


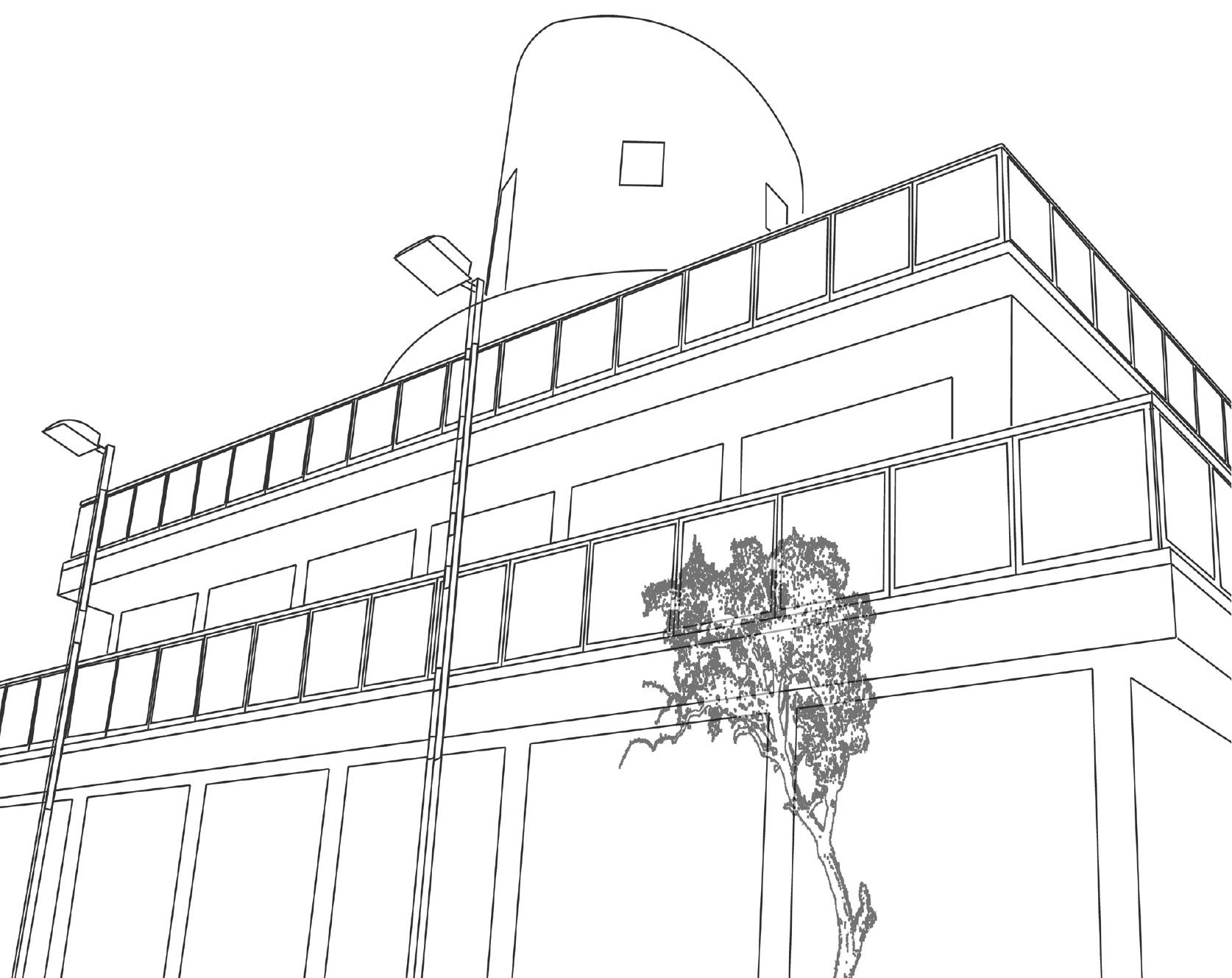

